\title{
Performance of Ultrasound in the Clinical Evaluation of Gout and Hyperuricemia
}

\author{
Ling Cao ${ }^{10},{ }^{1,2}$ Tianyi Zhao, ${ }^{1,2}$ Chunmei Xie, ${ }^{2,3}$ Shucong Zheng, ${ }^{1,2}$ Weiguo Wan, ${ }^{1,2}$ \\ Hejian Zou, ${ }^{1,2}$ and Xiaoxia Zhu $\mathbb{D}^{1,2}$ \\ ${ }^{1}$ Division of Rheumatology, Huashan Hospital, Fudan University, Shanghai, China 200040 \\ ${ }^{2}$ Institute of Rheumatology, Immunology, and Allergy, Fudan University, Shanghai, China 200040 \\ ${ }^{3}$ Ultrasonic Department, Huashan Hospital North, Fudan University, Shanghai, China 200040
}

Correspondence should be addressed to Xiaoxia Zhu; 15901811889@163.com

Received 13 February 2021; Revised 2 March 2021; Accepted 22 March 2021; Published 5 April 2021

Academic Editor: Lihua Duan

Copyright (C) 2021 Ling Cao et al. This is an open access article distributed under the Creative Commons Attribution License, which permits unrestricted use, distribution, and reproduction in any medium, provided the original work is properly cited.

\begin{abstract}
Objective. To evaluate monosodium urate (MSU) crystal deposition and related lesions in the joints of patients with gout and hyperuricemia (HUA) using ultrasound. To explore the association between ultrasound findings and clinical features in gout and HUA. Methods. A total of 202 patients with gout and 43 asymptomatic patients with HUA were included. The clinical data and ultrasonic assessment results were collected and statistically analyzed. Results. Deposition of MSU crystals was found in $25.58 \%(11 / 43)$ of patients with asymptomatic HUA and $76.24 \%(154 / 202)$ of patients with gout. Of the 1,082 joints from patients with gout examined, 33.09\% (358/1082) displayed MSU crystal deposition. In the joints with MSU crystal deposition, $77.37 \%$ (277/358) had a history of attacks. Among the joints of gouty arthritis, double contour sign (DCS), hyperechoic aggregate (HAG), and tophi were found in $32.65 \%$ (159/487), 7.80\% (38/487), and $24.64 \%(120 / 487)$ of the joints, respectively. DCS and tophi, but not HAG, increasingly appeared with the extension of gout duration. In patients with more than 15 years of gout history, DCS, Tophi, and HAG were found in $48.18 \%, 40.00 \%$, and $6.36 \%$ of US assessed joints, respectively. In patients with gout, synovial lesion and bone erosion were found in $17.74 \%(192 / 1082)$ and $7.58 \%(82 / 1082)$ of joints, respectively. The synovial lesion was related to HAG, while bone erosion was related to tophi and DCS. Nephrolithiasis was detected in $20.30 \%$ (41/202) of patients with gout and 4.65\% (2/43) of HUA patients, indicating that nephrolithiasis occurred in more patients with gout than in patients with HUA. Conclusion. HAG is an early performance of MSU crystal deposition in joints of gout and HUA. Both DCS and tophi are risk factors for bone erosion. Early urate-lowering therapy (ULT) should be considered in patients with gout, DCS, or tophi.
\end{abstract}

\section{Introduction}

Gout is a common inflammatory disease induced by the deposition of monosodium urate (MSU) crystals in joints and surrounding soft tissues, and hyperuricemia (HUA) is a critical factor for developing symptomatic gout. Most HUA patients do not have gouty arthritis, although MUS crystals are detected in their joints $[1,2]$. Chronic pain, soreness, or numbness in the joints is reported in some patients without convincing clinical evidence of a gouty attack, and it is difficult to differentiate gout from osteoarthritis or other chronic arthritis. Noninvasive imaging evidence of urate deposition in joints is valuable and helpful for differential diagnosis [3].

Ultrasound (US), a noninvasive, free of ionizing radiation, convenient, and inexpensive approach, has recently been used to identify MSU crystal deposits for diagnosing gout $[4,5]$. A standardized definition of ultrasound lesions with the elementary morphostructural changes in gout has been established in an international consensus [6]. Double contour sign (DCS), hyperechoic aggregates (HAG), and tophi are considered characteristic US abnormalities of MSU crystal deposits. Furthermore, synovial lesions (i.e., synovial hypertrophy and synovitis) and bone erosion are 
regularly detected by ultrasound in gout and HUA patients [2].

US findings are known to be crucial evidence for the diagnosis or differential diagnosis for gout $[7,8]$. The various US phenomena may indicate various joint injuries. Tophi detected by US is reported to be associated with worse foot pain and disability [9]. However, US evidence of MSU crystal deposition can be found in asymptomatic joints in gouty patients, while MSU crystals may not always be detected in the gouty joints with attacks [10]. Furthermore, joint US may show the sign of MSU crystal deposition in asymptomatic HUA and normal people [11]. These findings suggest that a reliable correlation between MSU crystal deposition and gouty attack or erosion remains unclear.

In this study, we retrospectively analyzed the joints' US results from patients who were diagnosed with gout or HUA to validate the US assessment of MSU crystal deposition and lesion in joints and evaluate the clinical value of US application in the diagnosis and prevention of gout and HUA.

\section{Methods}

2.1. Study Cohort and Methods. Patients aged 18-75 years, diagnosed with gout or asymptomatic HUA in a goutspecialized clinic in Huashan Hospital from Aug $1^{\text {st }}, 2016$, to Feb $28^{\text {th }}, 2019$, were eligible for this study. A total of 245 patients (202 with gout, 43 with asymptomatic HUA) were included. The required inclusion criteria for patients with gout matched the gout classification criteria of the American College of Rheumatology (ACR)/European League Against Rheumatism (EULAR) (2015). The exclusion criteria for patients with gout were prior diagnosis of other crystalrelated arthropathies, such as calcium pyrophosphate deposition disease with visible tophi. Urate levels in fasting serum in all $43 \mathrm{HUA}$ patients were greater than $420 \mu \mathrm{mol} / \mathrm{L}$, and this was confirmed at least twice. Besides the US results, all the following data, including demographics (i.e., sex, age, and disease duration), body mass index (BMI), and the clinical features of affected joints, were collected.

In the 202 patients with gout, 1,082 joints, including the first metatarsophalangeal joint (MTP1), ankle, knee, acrotarsium, elbow, wrist, and hand joints, were analyzed using US. In the 43 HUA patients, 256 joints were examined. Most of the joints were the three vulnerable pairs of lower joints (MTP1, ankle, and knee), and the others were the joints with mild clinical manifestation, including numb, slight pain, or discomfort. The rheumatologist decided which and how many joints underwent US examination based on clinical judgment (the joints with symptoms and potentially affected joints).

Ethical approval for the study was obtained from the Institutional Review Board for Human Studies at Huashan Hospital, Fudan University (approval number: 2012137). All the included patients had provided a fully informed written consent form before data collection.

2.2. US Assessment. The US examination was performed by skilled sonographers who had more than 10 years of experi- ence in the musculoskeletal US in Huashan Hospital. Aplio i900 color ultrasonic diagnostic apparatus (probe frequency $5-18 \mathrm{MHz}$ ) was used for US examination. According to the international consensus of the standardized definition of US gout lesion published by OMERACT (Outcome Measures in Rheumatology), US Gout Task Force was employed [6, 12], and MSU crystal deposition in joints was diagnosed based on DCS, hyperechoic aggregates (HAG), and tophi. Synovial hypertrophy, synovitis, and bone erosion were considered joint damage.

2.3. Statistical Analysis. The analysis was performed using SPSS 19.0 (IBM). All data were presented as mean \pm SD or proportions. Comparisons of baseline data between the two groups were tested for statistical significance using a $t$-test or one-way analysis of variance test with a least significant difference multiple comparison test. $p$ values $<0.05$ were considered significant. Pearson's correlation coefficient was calculated to examine correlations between variables.

\section{Results}

3.1. Demographics and Clinical Characteristics of the Study Population. Demographics and clinical characteristics are summarized in Table 1. Among the 202 patients with gout, 191 (94.55\%) were male. The average age was 46.90 years old. Among the 43 HUA patients, 31 (72.09\%) were male. The average age was 44.47 years old. BMI in both gout $(25.59 \pm 3.48)$ and HUA $(25.64 \pm 3.03)$ patients was higher than the normal value (18.5-23.9), and there was no significant difference between the two groups of patients. Serum uric acid (SUA) in patients with gout $(524.24 \pm 79.68)$ was higher than in HUA patients $(493.40 \pm 66.85)$, which might be due to the longer course of HUA in the gout group.

The urate crystal deposits in the kidney directly result in chronic urate nephropathy. In Table 1 , the creatinine clearance rate (Ccr) in patients with gout $(95.55 \pm 2.55 \mathrm{~mL} /$ minute) is lower than in HUA patients $(106.42 \pm 5.54 \mathrm{~mL} /$ minute). $\mathrm{Ccr}<80 \mathrm{ml} /$ minute was detected in $39.60 \%$ (80/202) of patients with gout and $20.93 \%$ (9/43) of HUA patients. Hyperlipidemia and hyperglycemia were known to coexist with HUA and gout. In our study, both fasting blood glucose (GLT) and triglyceride (TG) were found to be higher in patients with gout than in HUA patients (Table 1). In patients with gout, our results showed more common comorbidities, such as kidney damage and metabolic disorder.

3.2. Clinical Characteristics of Gouty Attacks in the Population with Gout. We further analyzed the relationship between ultrasound abnormalities signs and clinical manifestations in the gout patients (Table 2, Figure 1). There were a total of 531 joints, including 187 (35.22\%) of MTP1, 155 (29.19\%) of ankles, 100 (18.83\%) of knees, $45(8.47 \%)$ of acrotarsium, $21(3.95 \%)$ of hand joints, $12(2.26 \%)$ of the wrist, and $11(2.07 \%)$ of the elbow in the 202 patients who had clinical attacks. More right joints showed attacks than left ones, although there was no statistical significance. Of the 202, the headmost involved joints were $99(49.01 \%)$ on MTP1, 61 (31.20\%) on the ankle, 21(10.40\%) on acrotarsium, 
TABLE 1: Demographics and clinical characteristics of the study population.

\begin{tabular}{lccc}
\hline Variable & Gout & HUA & $p$ value \\
\hline Total number & 202 & 43 & - \\
Male/female & $191 / 11$ & $31 / 12$ & - \\
Age (years) & $46.90 \pm 14.82$ & $44.47 \pm 18.18$ & 0.35 \\
BMI $(\mathrm{kg} / \mathrm{m} 2)$ & $25.59 \pm 3.48$ & $25.64 \pm 3.03$ & 0.93 \\
SUA $(\mu \mathrm{mol} / \mathrm{L})$ & $524.24 \pm 79.68$ & 4 & $0.018^{*}$ \\
Ccr $(\mathrm{mL} / \mathrm{min})$ & $95.55 \pm 2.55$ & $106.42 \pm 5.54$ & $<0.0001$ \\
TG $(\mathrm{mmol} / \mathrm{L})$ & $2.91 \pm 1.42$ & $1.80 \pm 1.29$ & $0.0051^{*}$ \\
GLU (mmol/L) & $5.73 \pm 0.62$ & $4.92 \pm 1.68$ & $0.0031^{*}$ \\
Cholesterol $(\mathrm{mmol} / \mathrm{L})$ & $4.97 \pm 1.04$ & $4.70 \pm 0.65$ & 0.34 \\
\hline
\end{tabular}

HUA: asymptomatic hyperuricemia; BMI: body mass index; SUA: serum uric acid; Ccr: creatinine clearance rate; TG: triglyceride; GLU: fasting blood glucose; ${ }^{*}$ significantly difference $(p<0.05)$.

TABLE 2: Clinical characteristics of gouty attacks in the gout population.

\begin{tabular}{lcccc}
\hline Flared joint & Total & Left & Right & $p$ value \\
\hline MTP1 & $35.22 \%(187 / 531)$ & $16.20 \%(86 / 531)$ & $19.02 \%(101 / 531)$ & 0.148 \\
Ankle & $29.19 \%(155 / 531)$ & $13.93 \%(74 / 531)$ & $15.25 \%(81 / 531)$ & 0.496 \\
Knee & $18.83 \%(100 / 531)$ & $9.04 \%(48 / 531)$ & $9.79 \%(52 / 531)$ & 0.671 \\
Acrotarsium & $8.47 \%(45 / 531)$ & $4.71 \%(25 / 531)$ & $3.76 \%(20 / 531)$ & 0.399 \\
Hands & $3.95 \%(21 / 531)$ & $1.88 \%(10 / 531)$ & $2.07 \%(11 / 531)$ & $1.51 \%(8 / 531)$ \\
Wrist & $2.26 \%(12 / 531)$ & $0.75 \%(4 / 531)$ & $1.13 \%(6 / 531)$ & 0.220 \\
Elbow & $2.07 \%(11 / 531)$ & $0.94 \%(5 / 531)$ & & 1 \\
\hline
\end{tabular}

MTP1: first metatarsophalangeal joint.

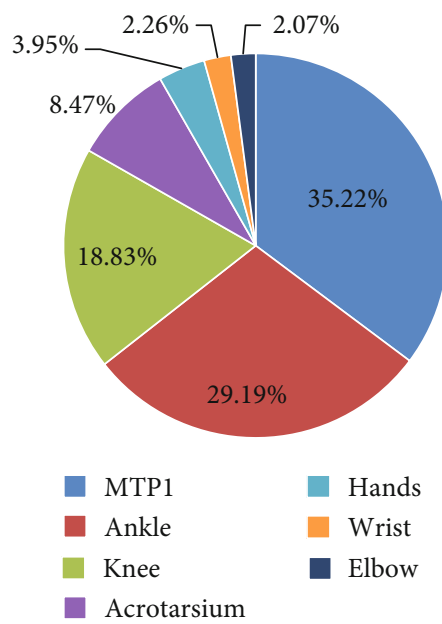

FIGURE 1: Clinical characteristics of gouty attacks in the gout population.

$14(6.93 \%)$ on the knee, $3(1.49 \%)$ on the wrist, and $4(1.98 \%)$ on hand joints. The results indicate that MTP1, ankle, and acrotarsium are more susceptible to be attacked.

3.3. Global US Findings in Patients with Gout and Hyperuricemia. In the 202 patients with gout, MSU crystals were detected in at least one of the joints in $76.24 \%$ (154/202), and $23.76 \%$ (48/202) of patients did not present any MSU crystals in the examined joints. MSU crystals were detected in 358 (33.09\%) joints of the 1,082 joints examined (Table 3(a)). Two-hundred and seventy-seven $(77.37 \%)$ of the 358 joints displayed MSU crystals and attacks. In the HUA patients, MSU crystals were detected in 11 joints among the 256 joints that underwent US examination, and the positive rate was $4.3 \%(11 / 256)$. Interestingly, these 11 joints belonged to 11 patients, and each patient had only one joint with positive US signs of MSU crystal deposition (Table 3(a)).

In the 1,082 joints of the patients with gout, 487 joints had at least one attack, while no attacks were reported in 541 joints (Table 3(b)). In the 487 joints, MSU crystals were found in $56.88 \%(277 / 487)$ using US. Among these joints, $32.65 \%$ (159/487), 7.80\% (38/487), and 24.64\% (120/487) were DCS, HAG, and tophi, respectively.

3.4. Synovial Lesion and Bone Erosion in the Patients with Gout. Besides the US signs of MSU crystal deposition, synovial lesions (i.e., synovial hypertrophy and synovitis) and bone erosion were regularly detected in the patients. In the patients with gout, synovial lesions were found in 192 (17.74\%) joints, and bone erosion was found in $82(7.58 \%)$ joints in 1,082 joints examined. In the 192 joints with synovial lesions, $24.48 \%$ (47/192), $11.98 \%$ (23/192), and $12.50 \%$ (24/192) were simultaneously positive for DCS, HAG, and tophi, respectively. In the 82 joints with bone erosion, $56.10 \%$ (46/82), 7.69\% (4/82), and 75.61\% (62/82) were simultaneously positive for DCS, HAG, and tophi, respectively (Table 4(a)). We further analyzed the correlation 
TABLE 3

(a) Global US findings in patients with gout and hyperuricemia

\begin{tabular}{lcccc}
\hline & \multicolumn{5}{c}{ Patients } \\
& Gout & HUA & $p$ value & $X^{2}$ \\
\hline Total patient & 202 & 43 & - & - \\
Positive patient* $^{*}$ & $154(76.24 \%)$ & $11(25.58 \%)$ & $<0.001$ & 41.369 \\
\hline \multicolumn{5}{c}{ Joints } \\
& Gout & HUA & $p$ value & $X^{2}$ \\
\hline Total detected joints & 1082 & 256 & - & \\
Positive joint & $358(33.09 \%)$ & $11(4.30 \%)$ & $<0.001$ & 85.913 \\
$\quad$ DCS & $228(21.07 \%)$ & $6(2.34 \%)$ & $<0.001$ & 50.320 \\
HAG & $64(5.91 \%)$ & $5(1.95 \%)$ & 0.04 & 4.168 \\
Tophi & $159(14.70 \%)$ & 0 & $<0.001$ & 42.693 \\
\hline
\end{tabular}

*Positive patient: MSU crystal deposits in at least one joint of the patient.

(b) US findings and clinical attacks in the joints of gouty patients

\begin{tabular}{lcc}
\hline & Ever attacked & Never attacked \\
\hline Total detected joints & 487 & 595 \\
Positive joints & $277 / 487(56.88 \%)$ & $67 / 595(11.26 \%)$ \\
DCS & $159 / 487(32.65 \%)$ & $43 / 595(7.23 \%)$ \\
HAG & $38 / 487(7.80 \%)$ & $16 / 595(2.69 \%)$ \\
Tophi & $120 / 487(24.64 \%)$ & $16 / 595(2.69 \%)$ \\
\hline
\end{tabular}

between synovial lesions and bone erosion with three US assessments. The results showed that synovial lesions were associated with HAG $(p<0.01)$ (Table $4(\mathrm{~b}))$, and bone erosion was associated with tophi $(p<0.001)$ and DCS $(p<0.01)$ (Table 4(c)).

3.5. Course Time and the MSU Crystals in Patients with Gout. The progression of gout can be defined by four pathophysiological stages: hyperuricaemia without evidence of monosodium urate crystal deposition or gout, crystal deposition without symptomatic gout, crystal deposition with acute gout flares, and advanced gout characterized by tophi, chronic gouty arthritis, and radiographic erosions. When the urate level in serum exceeds its saturation concentration, the precipitated urate crystals deposit in joints and soft tissues. In this study, we found that MSU crystal deposition was correlated with the SUA level $(p<0.01)$ and the duration $(p<0.01)$. Furthermore, many patients had no awareness of their SUA level before seeking clinical specialists. In 202 patients with gout, only $17.33 \%$ (35/202) of the patients came for treatment at their early stage (course $<1$ year). Most had more than one year of gout history (Table 5(a)).

In patients with gout, the proportion of US-positive signs of MSU crystal deposition gradually increased, especially DCS and tophi during gout. In patients with more than 15 years of gout history, DCS and tophi were detected in $48.18 \%$ and $40.00 \%$ of joints, respectively, whereas in patients with less than one year of gout history, DCS and tophi were found only in $6.29 \%$ and $5.03 \%$ of joints, respectively. HAG
TABLE 4

(a) Synovial lesion and bone erosion in the patients with gout

\begin{tabular}{lc}
\hline & Joints \\
& Gout \\
\hline Total detected joint & 1082 \\
\hline Synovial lesion & $192 / 1082(17.74 \%)$ \\
DCS & $47 / 192(24.48 \%)$ \\
HAG & $23 / 192(11.98 \%)$ \\
Tophi & $24 / 192(12.50 \%)$ \\
\hline Bone Erosion & $82 / 1082(7.58 \%)$ \\
DCS & $46 / 82(56.10 \%)$ \\
HAG & $4 / 82(7.69 \%)$ \\
Tophi & $62 / 82(75.61 \%)$ \\
\hline
\end{tabular}

(b) Synovial lesion is related to HAG

\begin{tabular}{lcc}
\hline Synovial lesion & Number & $p$ value \\
\hline DCS & 47 & 0.385 \\
HAG & 23 & $0.01^{*}$ \\
Tophi & 24 & 0.316 \\
\hline Pearson's chi-squared test, ${ }^{*}$ significantly difference $(p<0.05)$. &
\end{tabular}

(c) Bone erosion is related to tophi and DCS

\begin{tabular}{lcc}
\hline Bone Erosion & Number & $p$ value \\
\hline DCS & 46 & $0.01^{*}$ \\
HAG & 4 & 1 \\
Tophi & 62 & $0.001^{*}$ \\
\hline
\end{tabular}

Pearson's chi-squared test, * significantly difference $(p<0.05)$.

showed no notable rising as gout duration extension. HAG was found in $5.03 \%(8 / 159)$ of joints of the patients who had less than one year of gout course, and it was $6.36 \%$ (7/110) in patients with more than 15 years of gout course (Table 5(a)).

In 35 patients with an early stage (gout course was less than one year), 28 patients came to the clinic at their first gout attack, and the affected joints were MTP1 [13], ankles [6], knee [1], acrotarsium [2], and hand joints [2]. In the 17 affected MTP1 joints, eight (47.06\%) were positive for MSU crystal deposition (3 DCS, 3 HAG, 2 tophi, and 1 DCS + tophi) (Table 5(b)).

3.6. Correlation of Joint MSU Crystal Deposition with Nephrolithiasis. Patients with gout were reported to be prone to have nephrolithiasis, acute renal colic, and hematuria, although it is difficult to determine the type of crystal in the kidney. In this study, we further analyzed the US data of the kidney. Nephrolithiasis was defined as US signs of calculus or crystal deposition in the kidney. We found that nephrolithiasis was detected in $20.30 \%$ (41/202) of patients with gout and $4.65 \%(2 / 43)$ of HUA patients, indicating that nephrolithiasis occurred more commonly in patients with gout than in hyperuricemia patients. The findings also 
TABLE 5

(a) Clinical course and the MSU deposition in gout patients

\begin{tabular}{|c|c|c|c|c|c|}
\hline & \multicolumn{5}{|c|}{ Course (years) } \\
\hline & $<1$ & $1-5$ & $5-10$ & $10-15$ & $>15$ \\
\hline Patients & 35 & 70 & 50 & 28 & 19 \\
\hline Total detected joints & 159 & 380 & 283 & 150 & 110 \\
\hline Positive joints & $13.84 \%(22 / 159)$ & $20.79 \%(79 / 380)$ & $39.93 \%(113 / 283)$ & $48.00 \%(72 / 150)$ & $65.45 \%(72 / 110)$ \\
\hline DCS & $6.29 \%(10 / 159)$ & $12.89 \%(49 / 380)$ & $23.32 \%(66 / 283)$ & $33.33 \%(50 / 150)$ & $48.18 \%(53 / 110)$ \\
\hline HAG & $5.03 \%(8 / 159)$ & $4.74 \%(18 / 380)$ & $8.48 \%(24 / 283)$ & $4.67 \%(7 / 150)$ & $6.36 \%(7 / 110)$ \\
\hline Tophi & $5.03 \%(8 / 159)$ & $6.05 \%(23 / 380)$ & $14.49 \%(41 / 283)$ & $27.33 \%(41 / 150)$ & $40.00 \%(44 / 110)$ \\
\hline Synovial lesion & $12.58 \%(20 / 159)$ & $15.26 \%(58 / 380)$ & $19.08 \%(54 / 283)$ & $22.67 \%(34 / 150)$ & $23.64 \%(26 / 110)$ \\
\hline Bone erosion & 0 & $2.11 \%(8 / 380)$ & $6.36 \%(18 / 283)$ & $18 \%(27 / 150)$ & $26.36 \%(29 / 110)$ \\
\hline
\end{tabular}

(b) US assessment in patients at first acute attack

\begin{tabular}{|c|c|c|}
\hline \multirow{2}{*}{ Attacked joints [28]. } & \multicolumn{2}{|c|}{ MSU crystal deposition } \\
\hline & Total & US signs \\
\hline MTP1 [13] & 8 & 3 DCS; 3 HAG; 2 tophi; 1 DCS + tophi \\
\hline Ankle [6] & 2 & $1 \mathrm{DCS} ; 1 \mathrm{HAG}$ \\
\hline Knee [1] & 0 & 0 \\
\hline Acrotarsium [2] & 1 & $1 \mathrm{HAG}$ \\
\hline Hand joints [2] & 0 & 0 \\
\hline
\end{tabular}

TABLE 6: Correlation of joint MSU deposition with nephrolithiasis.

\begin{tabular}{lccc}
\hline Joint & Positive & Negative & Total \\
Kidney & 37 & 4 & 41 \\
\hline Positive & 117 & 44 & 161 \\
Negative & 154 & 48 & 404 \\
$\quad$ Total & & & \\
\hline
\end{tabular}

Pearson's chi-squared test, $X^{2}=5.571, p=0.018(p<0.05$ considered as significantly difference).

showed that nephrolithiasis was remarkably relevant to MSU crystal deposition in joints in $(p<0.05)$ (Table 6).

\section{Discussion}

The demographics of cohorts in this study show that the average ages of gout and HUA patients in this study were 46.90 and 44.47 years old, respectively, which were younger than the previously reported average age of 52.69 years from the HUA data in a Chinese national cross-sectional survey in 2014 [14]. This suggests that gout and HUA are increasing in the younger population due to lifestyle changes [15]. Our results are consistent with previous reports that high BMI, hyperlipidemia, and hyperglycemia are notably complicated with gout and HUA, especially gout [16]. Therefore, middle-aged males with certain metabolic syndromes should be listed in the attention-demanding high-risk population of gout and HUA.

Our data indicate that lower limb joints are more vulnerable in patients with gout. MTP1 (49.01\%), followed by the ankle $(31.20 \%)$ and acrotarsium $(10.40 \%)$, is the most affected joints. The upper limb joints, including hands, wrist, and elbow, are rarely involved. This result strongly supports the gout diagnostic value of MTP1 and ankle attacks, especially at an early stage.

For patients without classic symptoms, MSU crystal deposition could be identified by image-based examination, such as dual-energy computed tomography (DECT) and US. It has been reported that the MSU burden volume, which is predictive of the risk of flares [17], can be measured using DECT [13]. Due to being noninvasive, free of ionizing radiation, convenient, and inexpensive, US is more widely used in the clinic. Our study shows that MSU deposition can be detected by US, and the most frequently affected joint is MTP1 in the gout patients. This result is consistent with clinical findings reported by other groups [7].

Previous research suggested that MSU deposition in joints is a crucial factor of gouty arthritis attack [4]. In this study, 28 gout patients came to outpatients at their first acute attack and took a joint examination by US. In the 28 patients, only $39.29 \%(11 / 28)$ attacked joints were found with MSU deposition. Furthermore, among the total 11 gouty joints with initial attacks, large joints such as the knee and ankle were the majority of MSU deposition-positive joints. Only one MTP1 was positive of MSU crystal deposition detected by US.

Moreover, of the 17 patients who were most attacked on MTP1 at an early stage (course < 1 year), $47.06 \%(8 / 17)$ were positive for MSU crystal deposition in the MTP1. These results raise a serious question about how sensitive the US is to detect MSU crystal deposition in joints, particularly in smaller joints of the lower extremities at an early stage. Surprisingly, $77.37 \%$ of the patients with US-detected MSU 
crystal deposits in joints had gouty attacks in the past. Due to practical reasons, the selection of joints for US examination is based on rheumatologist personal judgment in this retrospective study, and a certain bias cannot be ruled out. This interpretation is consistent with previous research, as ultrasound features of MSU crystal deposition had a high positive predictive value but more limited sensitivity for early gout [18]. Further investigation is warranted to validate the US's sensitivity and specificity in detecting MSU crystal deposition in various joints.

According to "2018 updated European League Against Rheumatism evidence-based recommendations for the diagnosis of gout" [19], MSU crystal deposition is crucial evidence for gout diagnosis. In our study, DCS and tophi were frequently detected by US in gout patients, and they are notably increased with the disease history of gout. HAG was reported to be the most common sign at the early stage, especially in asymptomatic joints [20]. In an animal experiment where MSU was injected into the knees of rabbits, HAG was found frequently displayed in $75 \%$ of knees at an early stage (day 7 after MSU injection) [21]. Collectively, our data support that HAG is the US sign for early MSU crystal deposition, while DCS and tophi are a useful signature of MSU crystal deposition for chronic gout.

HAG was found to be associated with synovial lesions in this study. We found synovial lesion was notably detected at an early stage and gradually increased with the gout course extension. It has been reported that urate-lowering therapy (ULT) with febuxostat for 24 months reduced synovitis detected by MRI in patients with acute gout [22]. ULT may diminish HAG and improve synovitis and synovial hypertrophy. Bone erosion was known to be an irreversible injuriousness in gout [23]. In our study, tophi and DCS were both found to be correlated with bone erosion. Timely, ULT may minimize tophi and DCS and block bone injury in the gout patients.

Male, diabetes, obesity, low $\mathrm{pH}$ in urine, hyperuricosuria, and low urine volume have been reported to be the main etiologic factors of nephrolithiasis [24, 25]. Due to oversaturated urate and deposition of its crystals in the kidney, it is not surprising that a higher prevalence of nephrolithiasis is found in gout patients [26]. In this study, nephrolithiasis is remarkably relative to MSU crystal deposition in gouty arthritis. The result suggests that the patients with MSU crystal deposition in joints may be prone to suffer nephrolithiasis. Although MSU crystal deposition is related to high serum uric acid [27], systemic conditions such as genetic predisposition, geographical location, dietary indiscretion, and various metabolic characteristics should be considered as other risk factors [28, 29].

\section{Conclusion}

Ultrasound is a clinically convenient approach to detect MSU crystal deposits in joints for supporting the diagnosis of gout. HAG is considered an early sign of MSU crystal deposition in joints, whereas DCS and tophi correlate with bone erosion. Early ULT might be effective in the reduction of HAG and partially prevent synovitis and synovial hypertrophy. ULT should be considered when gout patients are detected with DCS or tophi in joints.

\section{Data Availability}

The datasets used and analyzed during the current study are available from the corresponding author on reasonable request.

\section{Disclosure}

The funders had no role in the study design, data collection and analysis, decision to publish, and manuscript preparation.

\section{Conflicts of Interest}

The authors declare that they have no competing interests.

\section{Authors' Contributions}

Ling Cao, and Tianyi Zhao contributed equally to this paper. Ling Cao contributed to the data collection and data acquisition. Tianyi Zhao contributed to the data interpretation. Chunmei Xie contributed to testing the patients. Shucong Zheng contributed to the experimental design. Weiguo Wan contributed to the computational data analysis. Hejian Zou and Xiao Xia Zhu contributed to the manuscript drafting and conceived the study. All authors contributed to the manuscript review. All authors read and approved the final manuscript. The manuscript's preprint is found in the link below: https://www.researchsquare.com/article/rs-108529/v1.

\section{Acknowledgments}

We also thank LetPub (http://www.letpub.com/) for its linguistic assistance during the preparation of this manuscript. This work was supported by the National Natural Science Foundation of China (No. 81302573; No. 81671588; No. 82071830), Research Funding from Shanghai Hospital Development Center (No. SHDC12016227; No. 16CR3012A), Medical Health Projects from BaoShan District, Shanghai (No. 18-E-27), and Shanghai Pujiang Young Rheumatologists Training Program (No. SPROG201902).

\section{References}

[1] S. Stewart, H. Maxwell, and N. Dalbeth, "Prevalence and discrimination of OMERACT-defined elementary ultrasound lesions of gout in people with asymptomatic hyperuricaemia: a systematic review and meta-analysis," Seminars in Arthritis and Rheumatism, vol. 49, no. 1, pp. 62-73, 2019.

[2] S. Stewart, N. Dalbeth, A. C. Vandal, B. Allen, R. Miranda, and K. Rome, "Ultrasound features of the first metatarsophalangeal joint in gout and asymptomatic hyperuricemia: comparison with normouricemic individuals," Arthritis care \& research, vol. 69, no. 6, pp. 875-883, 2017.

[3] L. Durcan, R. Grainger, H. I. Keen, W. J. Taylor, and N. Dalbeth, "Imaging as a potential outcome measure in gout studies: a systematic literature review," Seminars in Arthritis and Rheumatism, vol. 45, no. 5, pp. 570-579, 2016. 
[4] E. Naredo, J. Uson, M. Jiménez-Palop et al., "Ultrasounddetected musculoskeletal urate crystal deposition: which joints and what findings should be assessed for diagnosing gout?," Annals of the Rheumatic Diseases, vol. 73, no. 8, pp. 15221528, 2014.

[5] T. Neogi, T. L. Jansen, N. Dalbeth et al., "2015 gout classification criteria: an American College of Rheumatology/European league against rheumatism collaborative initiative," Annals of the Rheumatic Diseases, vol. 74, no. 10, pp. 1789-1798, 2015.

[6] M. Gutierrez, W. A. Schmidt, R. G. Thiele et al., "International consensus for ultrasound lesions in gout: results of Delphi process and web-reliability exercise," Rheumatology (Oxford, England), vol. 54, no. 10, pp. 1797-1805, 2015.

[7] P. Zufferey, R. Valcov, I. Fabreguet, A. Dumusc, P. Omoumi, and A. So, "A prospective evaluation of ultrasound as a diagnostic tool in acute microcrystalline arthritis," Arthritis Research \& Therapy, vol. 17, no. 1, p. 188, 2015.

[8] E. Norkuviene, M. Petraitis, I. Apanaviciene, D. Virviciute, and A. Baranauskaite, "An optimal ultrasonographic diagnostic test for early gout: a prospective controlled study," The Journal of International Medical Research, vol. 45, no. 4, pp. 14171429, 2017.

[9] S. Stewart, N. Dalbeth, A. C. Vandal, B. Allen, R. Miranda, and K. Rome, "Are ultrasound features at the first metatarsophalangeal joint associated with clinically-assessed pain and function? A study of people with gout, asymptomatic hyperuricaemia and normouricaemia," Journal of foot and ankle research, vol. 10, no. 1, p. 22, 2017.

[10] A. Di Matteo, E. Filippucci, E. Cipolletta et al., "Ultrasound and clinical features of hip involvement in patients with gout," Joint, Bone, Spine, vol. 86, no. 5, pp. 633-636, 2019.

[11] Q. Zhang, F. Gao, W. Sun, J. Ma, L. Cheng, and Z. Li, "The diagnostic performance of musculoskeletal ultrasound in gout: a systematic review and meta-analysis," PLoS One, vol. 13, no. 7, article e0199672, 2018.

[12] T. Cazenave, V. Martire, A. M. Reginato et al., "Reliability of OMERACT ultrasound elementary lesions in gout: results from a multicenter exercise," Rheumatology International, vol. 39, no. 4, pp. 707-713, 2019.

[13] T. Pascart, A. Grandjean, B. Capon et al., "Monosodium urate burden assessed with dual-energy computed tomography predicts the risk of flares in gout: a 12-month observational study," Arthritis Research \& Therapy, vol. 20, no. 1, p. 210, 2018.

[14] R. Liu, C. Han, D. Wu et al., "Prevalence of hyperuricemia and gout in mainland China from 2000 to 2014: a systematic review and meta-analysis," BioMed Research International, vol. 2015, Article ID 762820, 12 pages, 2015.

[15] J. H. Chen, C. P. Wen, S. B. Wu et al., "Attenuating the mortality risk of high serum uric acid: the role of physical activity underused," Annals of the Rheumatic Diseases, vol. 74, no. 11, pp. 2034-2042, 2015.

[16] C. Zhu, R. Cui, M. Gao et al., "The associations of serum uric acid with obesity-related acanthosis nigricans and related metabolic indices," International Journal of Endocrinology, vol. 2017, Article ID 5438157, 9 pages, 2017.

[17] D. Bhadu, S. K. Das, A. Wakhlu, U. Dhakad, and M. Sharma, "Ultrasonographic detection of double contour sign and hyperechoic aggregates for diagnosis of gout: two sites examination is as good as six sites examination," International Journal of Rheumatic Diseases, vol. 21, no. 2, pp. 523-531, 2018.
[18] A. Ogdie, W. J. Taylor, T. Neogi et al., "Performance of ultrasound in the diagnosis of gout in a multicenter study: comparison with monosodium urate monohydrate crystal analysis as the gold standard," Arthritis \& Rhematology, vol. 69, no. 2, pp. 429-438, 2017.

[19] P. Richette, M. Doherty, E. Pascual et al., “2018 updated European league against rheumatism evidence-based recommendations for the diagnosis of gout," Annals of the Rheumatic Diseases, vol. 79, no. 1, pp. 31-38, 2019.

[20] L. Zhu, S. Zheng, W. Wang, Q. Zhou, and H. Wu, "Combining hyperechoic aggregates and the double-contour sign increases the sensitivity of sonography for detection of monosodium urate deposits in gout," Journal of Ultrasound in Medicine, vol. 36, no. 5, pp. 935-940, 2017.

[21] C. Pineda, A. J. Fuentes-Gómez, C. Hernández-Díaz et al., "Animal model of acute gout reproduces the inflammatory and ultrasonographic joint changes of human gout," Arthritis Research \& Therapy, vol. 17, no. 1, p. 37, 2015.

[22] J. Collison, "Crystal arthritis: Febuxostat reduces synovitis in early gout," Nature Reviews Rheumatology, vol. 13, no. 12, p. 694, 2017.

[23] M. Wu, F. J. Liu, J. Chen et al., "Prevalence and factors associated with bone Erosion in patients with gout," Arthritis care \& research, vol. 71, no. 12, pp. 1653-1659, 2019.

[24] M. J. Roughley, J. Belcher, C. D. Mallen, and E. Roddy, "Gout and risk of chronic kidney disease and nephrolithiasis: metaanalysis of observational studies," Arthritis Research \& Therapy, vol. 17, no. 1, p. 90, 2015.

[25] A. J. Landgren, L. T. Jacobsson, U. Lindström et al., "Incidence of and risk factors for nephrolithiasis in patients with gout and the general population, a cohort study," Arthritis Research \& Therapy, vol. 19, no. 1, p. 173, 2017.

[26] T. Shimizu, H. Hori, M. Umeyama, and K. Shimizu, "Characteristics of gout patients according to the laterality of nephrolithiasis: a cross-sectional study using helical computed tomography," International Journal of Rheumatic Diseases, vol. 22, no. 4, pp. 567-573, 2019.

[27] A. Chhana, G. Lee, and N. Dalbeth, "Factors influencing the crystallization of monosodium urate: a systematic literature review," BMC Musculoskeletal Disorders, vol. 16, no. 1, p. 296, 2015.

[28] M. A. Martillo, L. Nazzal, and D. B. Crittenden, "The crystallization of monosodium urate," Current Rheumatology Reports, vol. 16, no. 2, p. 400, 2014.

[29] K. Sakhaee, "Epidemiology and clinical pathophysiology of uric acid kidney stones," Journal of Nephrology, vol. 27, no. 3, pp. 241-245, 2014. 